occurs after the dura has been opened, during retraction of the frontal lobe in order to gain free access to the sella turcica. In the transfrontal operation for orbital osteoma where the dura is not opened there would be no likelihood of injury to the olfactory bulb. In the case of orbital osteoma mentioned above smell was found to be normal after operation by the transfrontal route even though in that case the dura was of necessity opened for removal of the intracranial projection of the osteoma.

It is clear that decision on the problems of orbital osteoma which we have presented must await further experience. We would urge, however, that in cases of this nature all relevant methods of treatment should receive a careful trial. It is indeed regretable that any disease, such as this, should lie, through divisions and specialization of modern medicine, on the border line of three specialities. The only way to correct this state of affairs is to achieve the closest co-operation and to make combined studies of the type that we have attempted in this case.

\title{
BIBLIOGRAPHY.
}

1. Cushing, H.-Experiences with orbito-ethmoidal osteomata having intracranial complications. Surg. Gynaecol. and Obst., Vol. XLIV, p. 721, 1927.

2. Souttar, H. S.-Hunterian Lecture. New methods of surgical access to the brain. Brit. Med. Jl., Vol. I, p. 295, 1928.

3. Turner, A. Logan and Reynolds, F. Esmond.-A study of the paths of infection to the brain, meninges and venous blood sinuses from neighbouring peripheral foci of inflammation. III, Jl. of Laryngol. and Otol., Vol. XII, p. 717, 1926; V, Loc. ctt., Vol. XLII, p. 525, 1927.

\section{EMBOLISM OF THE ARTERIA CENTRALIS RETINAE}

\author{
BY \\ DR. NASR FARID BEY \\ CAIRO
}

IN August, 1930, I published in The British Journal of Ophthalmology (Vol. XIV, p. 402) an article on Spasm of the Central Artery. I have had the opportunity since of studying a case of embolism of the same artery. The purpose of this second communication is to compare the symptomatology of these two affections.

The most striking symptom of embolism of the central artery is sudden and complete blindness* of the eye in which the occlusion has occurred. If one examines such an eye immediately after the accident, one finds all the signs of a complete anaemia of the retina.

*Fuchs.-French Edition of 1906, p. 536. 
According to Abadie, embolism of the central artery is much less frequent than its spasmodic occlusion. The latter, due to a sclerosis of the artery, being always more or less gradual, the blindness appears periodically in the form of transitory attacks, whilst the blindness due to embolism is absolute and mostly definite. Further, it is comprehensible that, in embolism, the degeneration of the vessels is more pronounced than in spasm, as long as the latter is only intermittent. For the same reason the reaction of the pupil to light is, in embolism, absolutely lost, as a sign of the interruption of the reflex to direct light.

My observation, April 5, 1930.-A. B., a man aged 60 years: no history of previous diseases; no syphilis ; urine, normal.

Right eye. -6.0 D. sph. $\overline{\mathrm{c}}+0.5 \mathrm{D}$. cyl. $90^{\circ}=6 / 9$.

Sclerosis of the lens, but fundus is normal.

Left eye.-Sclerosis of the lens, but absolute blindness, without any direct light-reaction. Fundus examination shows all signs of complete anaemia of the retina.

Another oculist performed paracentesis of the left (blind) eye, and prescribed iodine--naturally without any result.

On April 18, I saw the patient again and examined him thoroughly in the laboratory of the Ophthalmic Hospital in

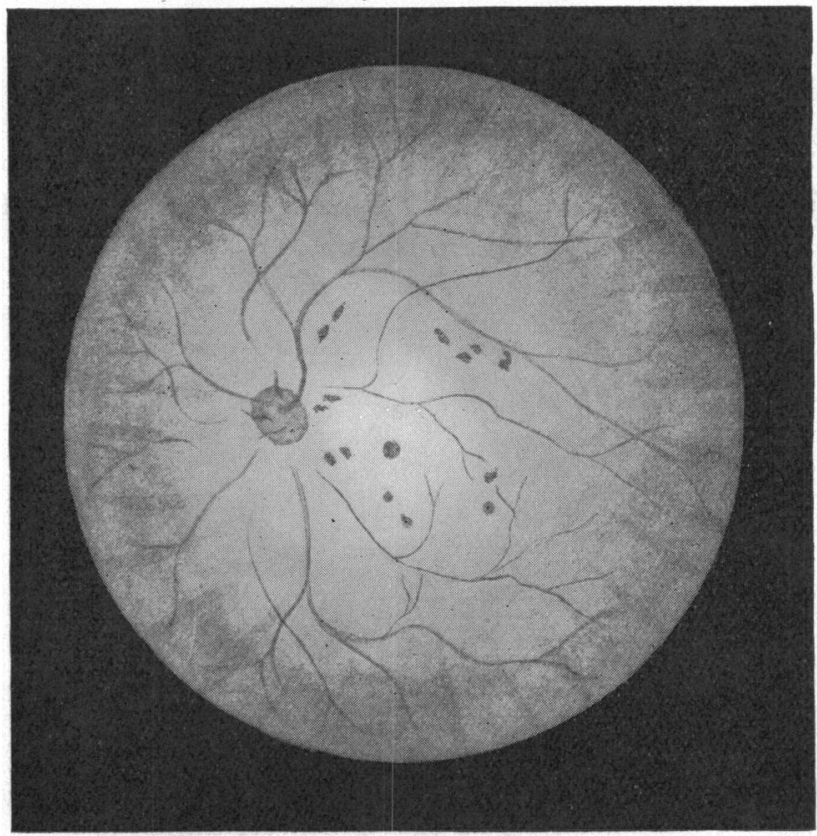

LEFT EYE. 
Ghizeh : the arteries of the fundus were like fine threads, and they seemed to be interrupted, especially on the temporal side.

On May 25, the patient showed increase of the blood-pressure, up to $260 \mathrm{~mm} . \mathrm{Hg}$. Treatment : Iodine, laxatives; milk and fruit diet.

In June the blood-pressure dropped to 210, but I found 10 per cent. albumin in the urine, with hyaline casts. Urea in the blood$13 \mathrm{mgm}$. only. The Wassermann reaction remained negative, even after one injection of neo-salvarsan.

Till to-day, the state of the eye is unchanged; a further careful examination with Gullstrand's ophthalmoscope showed nothing new.

In the beginning of November, 1930, the heart conditions were, according to Prof. Schrumpf Pierron, the following : Severe, still active aortitis with slight enlargement of the left heart and high blood-pressure $(140 / 220)$. The embolus was very probably due to a parietal thrombosis of the atheromatous aorta. No peripheral arteriosclerosis.

Conclusion:-Case of embolic occlusion of the central artery, with sudden blindness. Naturally there was no success as a result of any treatment, whilst in spasmodic occlusion due to progressive sclerosis of the artery, there is often a chance of recovery by aid of early and energetic treatment.

\section{ANNOTATION}

\section{"Following up"}

Under the heading of "Wasted effort in the Clinics" we find the following depressing statement in the American Journal of Ophthalmology for May 1931. " Forty cases of corneal ulcer (ordinary abrasions not being included), 16 cases of interstitial keratitis, 33 cases of phlyctenular keratoconjunctivitis, 21 cases of iritis, 54 cases of disease of the choroid and retina, six cases of papillitis, two cases of papilloedema, 26 cases of optic atrophy, and 12 cases of glaucoma made but a single visit each." One wonders whether similar figures would be found in English Outpatient Departments. One's impression is that they would not, but it is an investigation that might well be worth while carrying out, and the result might easily be surprising. One obvious fallacy would have to be borne in mind, however, namely, that outpatients frequently transfer themselves from one hospital to another, particularly when they have been given unpalatable advice, such as removal of an eye for a suspected malignant growth. The writer has seen many cases in his clinic who have 